\title{
Detection, Synchronization, and Doppler Scale Estimation with Multicarrier Waveforms in Underwater Acoustic Communication
}

\author{
Sean Mason, Christian R. Berger, Shengli Zhou, and Peter Willett \\ Department of Electrical and Computer Engineering, University of Connecticut, Storrs, Connecticut, 06269
}

\begin{abstract}
We propose a novel method for detection, synchronization, and Doppler scale estimation for underwater acoustic communication using orthogonal frequency division multiplex (OFDM) waveforms. The method involves transmitting two identical OFDM symbols together with a cyclic prefix, while the receiver uses a bank of parallel self-correlators matched to different Doppler scaling factors on waveform dilation or compression. We characterize the receiver operating characteristic in terms of probability of false alarm and probability of detection, and analyze the impact of Doppler scale estimation accuracy on the data transmission performance. We have tested the proposed method with real data from an experiment at Buzzards Bay, MA, Dec. 15, 2006. Using only one OFDM preamble, the proposed method achieves performance similar to an existing method that uses two linearly-frequency-modulated (LFM) waveforms, one as a preamble and the other as a postamble. Avoiding the need of buffering the whole data packet before data demodulation, the proposed method enables online receiver operation.
\end{abstract}

\section{INTRODUCTION}

Offline data processing based on recorded experimental data is often used to examine various underwater transmission schemes. Towards the development of an online underwater acoustic receiver, detection and synchronization are important, yet often overlooked tasks.

Synchronization typically entails transmission of a known preamble prior to the data, which can be easily detected by the receiver. Existing preambles in underwater telemetry are almost exclusively based on linearly frequency modulated (LFM) signals, also known as Chirp signals [1]. The receiver usually deploys a matched filter to synchronize the known template with the signal coming from one strong path, while suppressing other interfering paths. This approach has the following shortcomings: first, the noise level at the receiver has to be constantly estimated to achieve a constant false alarm rate (CFAR), usually accomplished using order statistics; second, its performance will degrade in the presence of dense and unknown multipath channels.

In this paper, we propose the use of a preamble that consists of two identical orthogonal frequency division multiplexing (OFDM) symbols preceded by a cyclic prefix (CP) for underwater acoustic (UWA) communications. This training pattern

The work of S. Mason and S. Zhou is supported by ONR YIP grant N0001407-1-0805, NSF grants ECS-0725562, and CNS-0721834. The work of C. R. Berger and P. Willett is supported by ONR grants N00014-07-1-0429 and N00014-07-1-0055. has been studied extensively in wireless OFDM systems for radio channels, see e.g., [2], [3], and has been included in the IEEE 802.11a/g standards [4]. The receiver effectively correlates the received signal with a delayed version of itself, since, thanks to the $\mathrm{CP}$ structure, the repetition pattern persists even in the presence of unknown multipath channels [2]. However, the synchronization algorithms that work in wireless radio channels will not perform well in UWA channels with large waveform expansion or compression, as the repetition period changes to an unknown value.

We develop a novel method that utilizes OFDM waveforms for detection, synchronization, and Doppler scale estimation. The receiver uses a bank of parallel branches, with each branch having a self-correlator matched to a different repetition period. Detection is declared when any of the branches leads to a correlation metric larger than a threshold. The branch with the largest metric yields a Doppler scale estimate and a coarse synchronization point. We characterize the receiver operating characteristics (ROC), and analyze the impact of Doppler scale estimation accuracy on the data demodulation performance. Compared with the LFM based alternative, the proposed approach has the following advantages: (1) the detection threshold can be preset and does not depend on the operating SNR; (2) the detection performance is based on the signal energy from all paths rather than only a single path; (3) it enables accurate Doppler scale estimation.

We have tested the proposed method with simulation and real data from an experiment at Buzzards Bay, MA, Dec. 15 , 2006. Using only one OFDM preamble, the proposed method achieves similar performance on the Doppler scale estimation accuracy and the bit error rate as the method based on the LFM pre- and post-ambles. However, the proposed method avoids the need to buffer the whole data packet before demodulation, which enables online receiver operation for multicarrier underwater acoustic communication.

The rest of this paper is as follows. The system model is presented in Section II, and the proposed receiver algorithm is described in Section III. Detection performance is derived in Section IV, and analysis of the impact of Doppler scale mismatch on the data demodulation performance is carried out in Section V. Section VI contains performance results, and Section VII contains the conclusion. 


\section{SySTEM MOdEL}

Zero-padded (ZP) OFDM is preferred for data transmission to avoid power consumption on the guard intervals [5], [6], [7]. For synchronization purposes, the preamble consists of two identical OFDM symbols together with a cyclic prefix. The overall transmission structure is shown in Fig. 1. The OFDM parameters can be selected independently for the preamble and data portions.

Suppose that $K_{0}$ subcarriers have been used in the preamble, and one OFDM symbol is of duration $T_{0}$. The subcarrier spacing is then $1 / T_{0}$ and the bandwidth is $B=K_{0} / T_{0}$. Let $f_{c}$ denote the carrier frequency, and $f_{k}=f_{c}+k / T_{0}$ denote the frequency for the $k$ th subcarrier in passband, where $k \in \mathcal{S}=\left\{-K_{0} / 2, \ldots, K_{0} / 2-1\right\}$. Let $T_{\mathrm{cp}}$ denote the CP length, and define a rectangular window of length $T_{\mathrm{cp}}+2 T_{0}$ as

$$
q(t)= \begin{cases}1 & t \in\left[-T_{\mathrm{cp}}, 2 T_{0}\right] \\ 0 & \text { otherwise. }\end{cases}
$$

The preamble in baseband can be written as

$$
x(t)=\sum_{k \in \mathcal{S}} s[k] e^{j 2 \pi \frac{k}{T_{0}} t} q(t),
$$

where $s[k]$ is the transmitted symbol on the $k$ th subcarrier. The passband signal is $\tilde{x}(t)=\operatorname{Re}\left\{e^{j 2 \pi f_{c} t} x(t)\right\}$.

The channel impulse response for a time-varying multipath underwater acoustic channel can be described by

$$
c(\tau, t)=\sum_{p} A_{p}(t) \delta\left(\tau-\tau_{p}(t)\right),
$$

where $A_{p}(t)$ is the path amplitude and $\tau_{p}(t)$ is the timevarying path delay. As in [7], we assume that all paths have a similar Doppler scaling factor $a$,

$$
\tau_{p}(t) \approx \tau_{p}-a t,
$$

and that the path gains $A_{p}$, the transmission delay $\tau_{p}$ and the Doppler scale $a$ are constant over the duration of the preamble. When these assumptions do not hold true, part of the useful signal is treated as additive noise, which increases the overall noise variance.

When the passband signal $\tilde{x}(t)$ goes through the channel described in (3) and (4), we receive:

$$
\begin{aligned}
\tilde{y}(t)= & \operatorname{Re}\left\{\sum_{k \in \mathcal{S}} s[k] e^{j 2 \pi f_{k}(1+a) t}\right. \\
& \left.\times \sum_{p} A_{p} q\left((1+a) t-\tau_{p}\right) e^{-j 2 \pi f_{k} \tau_{p}}\right\}+\tilde{n}(t),
\end{aligned}
$$

\begin{tabular}{|c|c|c|c|c|c|c|}
\hline \multicolumn{3}{|c|}{ preamble } & \multicolumn{4}{|c|}{ data transmission } \\
\hline $\mathrm{CP}$ & $\mathbf{x}$ & $\mathbf{x}$ & guard & ZP OFDM & --- & ZP OFDM \\
\hline
\end{tabular}

where $\tilde{n}(t)$ is the additive noise. Define $\tau_{\max }=\max _{p} \tau_{p}$, which is usually less than the $\mathrm{CP}$ length $T_{\mathrm{cp}}$. Using the definition of $q(t)$ in (1), we obtain

$$
\begin{aligned}
\tilde{y}(t)=\operatorname{Re}\left\{\sum_{k \in \mathcal{S}} H_{k} s[k] e^{j 2 \pi f_{k}(1+a) t}\right\}+\tilde{n}(t), \\
t \in \mathcal{T}_{\text {cyclic }}=\left[-\frac{T_{\mathrm{cp}}-\tau_{\max }}{1+a}, \frac{2 T_{0}}{1+a}\right],
\end{aligned}
$$

Fig. 1. A preamble, consisting of two identical OFDM symbols and a cyclic prefix, precedes the data transmission which uses zero padded OFDM.

where $H_{k}$ is the channel frequency response at $k$ th subcarrier:

$$
\begin{aligned}
H_{k} & =C\left(f_{k}\right), \\
C(f) & =\sum_{p} A_{p} e^{-j 2 \pi f \tau_{p}} .
\end{aligned}
$$

Converting the passband signal $\tilde{y}(t)$ to baseband, such that $\tilde{y}(t)=\operatorname{Re}\left\{y(t) e^{j 2 \pi f_{c} t}\right\}$, we have:

$$
y(t)=\sum_{k \in \mathcal{S}} H_{k} s[k] e^{j 2 \pi\left(\frac{k}{T_{0}}+a f_{k}\right) t}+n(t), \quad t \in \mathcal{T}_{\text {cyclic }},
$$

where $n(t)$ is the noise at baseband.

\section{The Proposed Algorithm}

Our proposed approach is to use a bank of self-correlators, with each matched to a different periodicity, as shown in Fig. 2. Detection, synchronization, and Doppler scale estimation are accomplished based on the correlation metrics from the bank of self-correlators.

The receiver operates on discrete samples. The baseband signal $y(t)$ is usually oversampled at a multiple of the system bandwidth with the sampling interval $t_{s}=1 /(\lambda B)$ :

$$
y[n]=\left.y(t)\right|_{t=n t_{s}} .
$$

The proposed algorithm includes the following steps:

S1: Each of the $L$ branches calculates a correlation metric with one candidate window size $N_{l}$, for each delay $d$,

$$
M\left(N_{l}, d\right)=\frac{\sum_{i=d}^{d+N_{l}-1} y^{*}[i] y\left[i+N_{l}\right]}{\sqrt{\sum_{i=d}^{d+N_{l}-1}|y[i]|^{2} \cdot \sum_{i=d}^{d+N_{l}-1}\left|y\left[i+N_{l}\right]\right|^{2}}},
$$

for $l=1, \ldots L$. The window size $N_{l}$ shall be close to $\lambda K_{0}$, which is the number of samples of one OFDM symbol when no Doppler scaling occurs.

S2: A detection is declared if the correlation metric of any branch exceeds a preset threshold $\Gamma_{\text {th }}$ :

$$
H_{1} \quad \text { if: } \quad \max _{l}\left|M\left(N_{l}, d\right)\right| \geq \Gamma_{\text {th }}
$$

Since the norm of the metric in (11) is between 0 and 1 , the threshold $\Gamma_{\text {th }}$ takes a value from $[0,1]$.

S3: The branch with the largest correlation metric is viewed as having the best match on the repetition period. Since Doppler scaling changes the period $T_{0}$ to $T_{0} /(1+a)$, the Doppler scale factor is estimated as

$$
\hat{a}=\frac{\lambda K_{0}}{\hat{N}}-1, \quad \text { where } \hat{N}=\arg \max _{\left\{N_{l}\right\}}\left|M\left(N_{l}, d\right)\right| .
$$




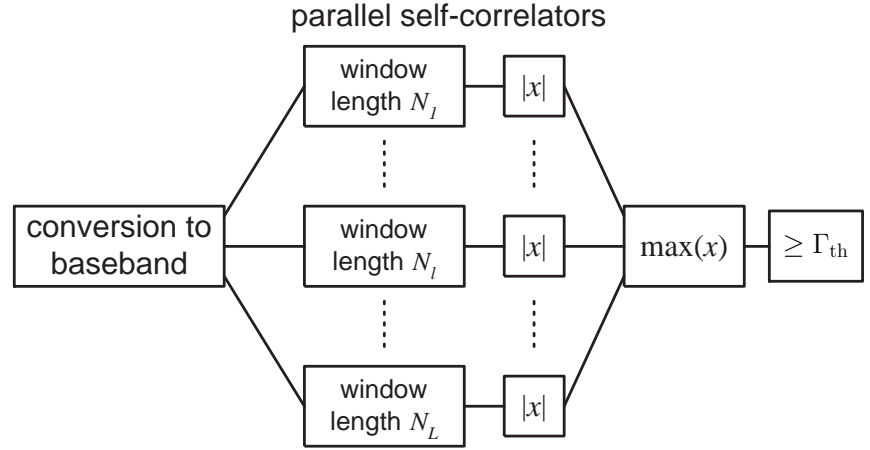

Fig. 2. To compensate for the time compression/dilation, multiple parallel branches are used, each tuned to a certain period $N_{l}$.

The relative speed between the transmitter and the receiver is estimated as

$$
\hat{v}=c \hat{a},
$$

where $c$ is the speed of sound in water. Additional processing can be used to refine the Doppler scale estimate, as will be shown later on in Section VI.

S4: Synchronization is performed on the branch that yields the maximum correlation metric. After the maximum is determined, the start of transmission is selected as in [2]; starting from the peak the $80 \%$ "shoulders" are found (first sample of this correlator branch before and after the peak that is less than $80 \%$ of the peak) and the middle is chosen as the synchronization point. This is beneficial, since due to the $\mathrm{CP}$ structure the correlation metric has a plateau around the peak [2].

So far, we have described the general procedure of the proposed detection, synchronization, and Doppler scale estimation algorithm, while some parameters are left to be specified. Important questions include:

- How to set the detection threshold?

- How many parallel branches are needed? What is the desired Doppler scale resolution?

We next address these questions by analyzing the detection performance in Section IV, and the data demodulation performance under Doppler scale mismatch in Section V.

\section{RECEIVER OPERATING CHARACTERISTICS}

We now analyze the false alarm and detection probabilities of a single branch, as a function of the threshold $\Gamma_{\text {th }}$ and the Doppler scale $a$. This will give us an understanding of the necessary Doppler scale spacing in the parallel self-correlator structure for detection purposes. Due to over-sampling, the summations in the metric given in (11) of the $l$ th branch can be well approximated with continuous time integrals:

$$
M(\hat{T}, t)=\frac{\int_{t}^{t+\hat{T}} y(\tau)^{*} y(\tau+\hat{T}) d \tau}{\sqrt{\int_{t}^{t+\hat{T}}|y(\tau)|^{2} d \tau \cdot \int_{t}^{t+\hat{T}}|y(\tau+\hat{T})|^{2} d \tau}},
$$

where

$$
\hat{T}=N_{l} \cdot t_{s}=\frac{T_{0}}{1+\hat{a}}, \quad t=d \cdot t_{s} .
$$

\section{A. Probability of False Alarm}

When no signal is present, $y(t)=n(t)$. Since $B \hat{T} \approx K_{0}$, we can find a set of $K_{0}$ orthonormal basis functions defined over a period of $T_{0}$. Define $\mathbf{n}_{t}$ and $\mathbf{n}_{t+\hat{T}}$ as the coefficient vectors of the continuous waveforms $n(\tau), \tau \in[t, t+\hat{T}]$ and $n(\tau), \tau \in[t+\hat{T}, t+2 \hat{T}]$ projected to the basis functions. Define their normalized versions as $\tilde{\mathbf{n}}_{t}=\mathbf{n}_{t} /\left\|\mathbf{n}_{t}\right\|$ and $\tilde{\mathbf{n}}_{t+\hat{T}}=\mathbf{n}_{t+\hat{T}} /\left\|\mathbf{n}_{t+\hat{T}}\right\|$. The correlator output (15) can be simplified to the inner product between two unit-length vectors as

$$
M(\hat{T}, t)=\tilde{\mathbf{n}}_{t}^{\mathcal{H}} \tilde{\mathbf{n}}_{t+\hat{T}} .
$$

Finding the probability of false alarm can now be linked to the Grassmannian line packing problem in [8]. Specifically, $\tilde{\mathbf{n}}_{i}$ can be viewed as coordinates of a point on the surface of a hypersphere with unit radius, centered around the origin. Since $n(t)$ is additive, white and Gaussian, $\tilde{\mathbf{n}}_{t}$ and $\tilde{\mathbf{n}}_{t+\hat{T}}$ are uniformly distributed on the surface of the hypersphere. Skipping some intermediate steps, which are available in [9], we obtain the probability of false alarm as:

$$
P_{f a}=\operatorname{Pr}\left(M(\hat{T}, t)>\Gamma_{\text {th }}\right)=\left(1-\Gamma_{\text {th }}^{2}\right)^{K_{0}-1} .
$$

Note that $P_{f a}$ does not depend on the power of the additive noise. Hence the threshold $\Gamma_{\text {th }}$ can be preset to achieve a constant false alarm rate independent of the noise level.

\section{B. Probability of Detection}

In [2], the absolute value of the correlation metric in the presence of the signal has been approximated as a Gaussian random variable to derive some approximate results. We would like to follow the same principle here. To this end, we propose to approximate the mean of the correlator output as:

$$
\mathrm{E}\{|M(\hat{T}, t)|\} \approx \frac{\alpha \gamma}{\gamma+1}
$$

where $\gamma$ is the signal-to-noise-ratio (SNR) at the receiver, and $\alpha$ is a constant that depends on the Doppler scaling. The variance is approximately [2]:

$$
\operatorname{Var}\{|M(\hat{T}, t)|\} \approx \frac{2 \gamma^{3}+5 \gamma^{2}+3 \gamma+1}{2 K_{0}(\gamma+1)^{4}} .
$$

In the radio channel case $|\alpha|=1$ and the variation is only due to the additive noise. In the underwater channel case, the constant $\alpha$ is a function of the channel realization and the Doppler scale mismatch $(a-\hat{a})$ (details can be found in [9]):

$$
\alpha \approx \frac{\left.\left|\sum_{k \in \mathcal{S}}\right| H_{k}\right|^{2} e^{-j 2 \pi k(a-\hat{a})} \mid}{\sum_{k \in \mathcal{S}}\left|H_{k}\right|^{2}} .
$$

Hence, an approximate expression for the probability of detection is

$$
P_{d}=\operatorname{Pr}\left\{|M(\hat{T}, t)| \geq \Gamma_{\text {th }}\right\} \approx Q\left(\frac{\Gamma_{\text {th }}-\mu_{\alpha}}{\sigma_{\alpha}}\right),
$$

where $Q(x)=(1 / \sqrt{2 \pi}) \int_{x}^{\infty} e^{-t^{2} / 2} d t$. 


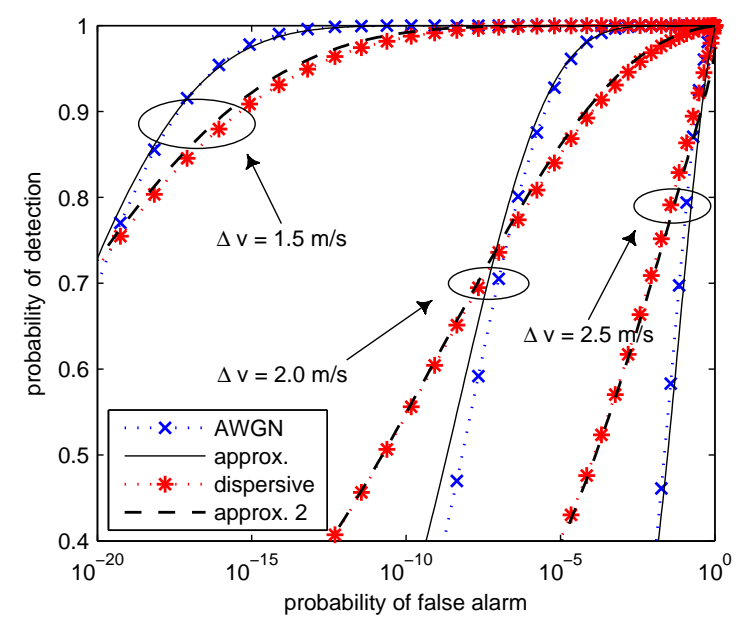

Fig. 3. ROC of the detection scheme for different Doppler scale mismatches $a-\hat{a}=\Delta v / c$, where $K_{0}=512, T_{0}=42.7 \mathrm{~ms}, B=12 \mathrm{kHz}$, and $\gamma=0 \mathrm{~dB}$; plotted are the Gaussian approximation of the probability of detection and simulated probability of detection for different channels against the exact probability of false alarm.

\section{Numerical Validation}

To assess the effect of Doppler scale mismatch on the detection performance, we simulate using the following steps:

1) Generate the baseband samples via (9) and (10).

2) Compare the statistics of the correlator output at the signal start $t=0$ on the branch with $N_{l}=\lambda K_{0}$.

To evaluate the performance of a time-dispersive fading channel, the expression in (21) is averaged over different channel realizations. We choose a channel profile which loses about 20 $\mathrm{dB}$ within $10 \mathrm{~ms}$, with independent complex Gaussian channel taps at the baseband.

We plot the ROC in Fig. 3. For both AWGN and dispersive channels, we observe that the simulation results match well the Gaussian approximation for the chosen speeds and SNR. The detection performance is superb as long as the speed mismatch is less than about $1.5 \mathrm{~m} / \mathrm{s}$.

\section{IMPACT OF DOPPlER SCAlE Estimation ACCURACY}

We now analyze the performance degradation on data transmission due to Doppler scale mismatch. For data transmission, we use ZP-OFDM, whose parameters can be chosen independently of the preamble. Let $T$ denote the symbol duration, $T_{g}$ the guard interval, $K$ the number of subcarriers. The subcarrier spacing is then $1 / T$ and the subcarrier frequencies are $f_{k}=f_{c}+k / T, k=-K / 2, \ldots, K / 2-1$. The received signal can be expressed as in (5) but with $q(t)$ replaced by the zero padding operator $g(t)=1, t \in[0, T]$.

A two-step approach to mitigating the channel Doppler effect was proposed in [7]. The first step is to resample $\tilde{y}(t)$ in the passband with a resampling factor $b$, leading to

$$
\tilde{z}(t)=\tilde{y}\left(\frac{t}{1+b}\right) .
$$

The baseband version of $\tilde{z}(t)$ is

$$
\begin{aligned}
z(t) & =e^{j 2 \pi \frac{a-b}{1+b} f_{c} t} \sum_{k \in \mathcal{S}} s[k] e^{j 2 \pi \frac{1+a}{1+b} \frac{k}{T} t} \\
& \times\left[\sum_{p} A_{p} e^{-j 2 \pi f_{k} \tau_{p}} g\left(\frac{1+a}{1+b} t-\tau_{p}\right)\right]+n(t) .
\end{aligned}
$$

The second step is to perform fine Doppler shift compensation on $z(t)$ to obtain $z(t) e^{-j 2 \pi \epsilon t}$, where $\epsilon$ is the estimated Doppler shift. Performing ZP-OFDM demodulation, the output $y_{m}$ on the $m$ th subchannel is

$$
z_{m}=\frac{1}{T} \int_{0}^{T+T_{g}} z(t) e^{-j 2 \pi \epsilon t} e^{-j 2 \pi \frac{m}{T} t} d t .
$$

Carrying out the integration, we obtain:

$$
z_{m}=C\left(\frac{1+b}{1+a}\left(f_{m}+\epsilon\right)\right) \sum_{k \in \mathcal{S}} s[k] \varrho_{m, k}+v_{m},
$$

where $v_{m}$ is the additive noise, $C(f)$ is defined in (8), and

$$
\begin{aligned}
\varrho_{m, k} & =\frac{1+b}{1+a} \cdot \frac{\sin \left(\pi \beta_{m, k} T\right)}{\pi \beta_{m, k} T} e^{j \pi \beta_{m, k} T}, \\
\beta_{m, k} & =(k-m) \frac{1}{T}+\frac{(a-b) f_{m}-(1+b) \epsilon}{1+a} .
\end{aligned}
$$

The effective SNR on the $m$ th subcarrier is

$$
\gamma_{m}=\frac{\left|\varrho_{m, m}\right|^{2} \sigma_{s}^{2}}{\frac{\sigma_{v}^{2}}{\left|C\left(\frac{1+b}{1+a}\left(f_{m}+\epsilon\right)\right)\right|^{2}}+\sum_{k \neq m}\left|\varrho_{m, k}\right|^{2} \sigma_{s}^{2}},
$$

where $\sigma_{s}^{2}=E\left[|s[k]|^{2}\right]$ and $\sigma_{v}^{2}=E\left[\left|v_{m}\right|^{2}\right]$. Ignoring the additive noise, the SNR is bounded by

$$
\gamma_{m} \leq \bar{\gamma}_{m}:=\frac{\left|\varrho_{m, m}\right|^{2}}{\sum_{k \neq m}\left|\varrho_{m, k}\right|^{2}}
$$

due to self-interference induced by Doppler scale mismatch.

We now evaluate the SNR uppperbound for two cases:

- Case 1: No Doppler shift compensation by setting $\epsilon=0$.

- Case 2: Ideal Doppler shift compensation where

$$
\epsilon_{\mathrm{opt}}=\frac{a-b}{1+b} f_{c}
$$

such that

$$
\beta_{m, k}=(k-m) \frac{1}{T}+\frac{(a-b) m / T}{1+a} .
$$

We set $f_{c}=27 \mathrm{kHz}, B=12 \mathrm{kHz}, K=1024, T=85.3$ $\mathrm{ms}$, and numerically evaluate the upperbounds $\bar{\gamma}_{m}$ for $\epsilon=0$ and $\epsilon=\epsilon_{\text {opt }}$. Fig. 4 shows the bounds for these two cases respectively, where the speed mis-match after resampling is $\Delta v \approx(a-b) \cdot c$. To limit the self-noise to be at least $20 \mathrm{~dB}$ below the signal power, we need $\Delta v$ to be less than $0.06 \mathrm{~m} / \mathrm{s}$ for $\epsilon=0$, while $\Delta v$ can be as large as $0.2 \mathrm{~m} / \mathrm{s}$ for $\epsilon=\epsilon_{\mathrm{opt}}$. Hence, the fine Doppler compensation step proposed in [7] improves the system performance substantially in the presence of Doppler scale mismatch. 


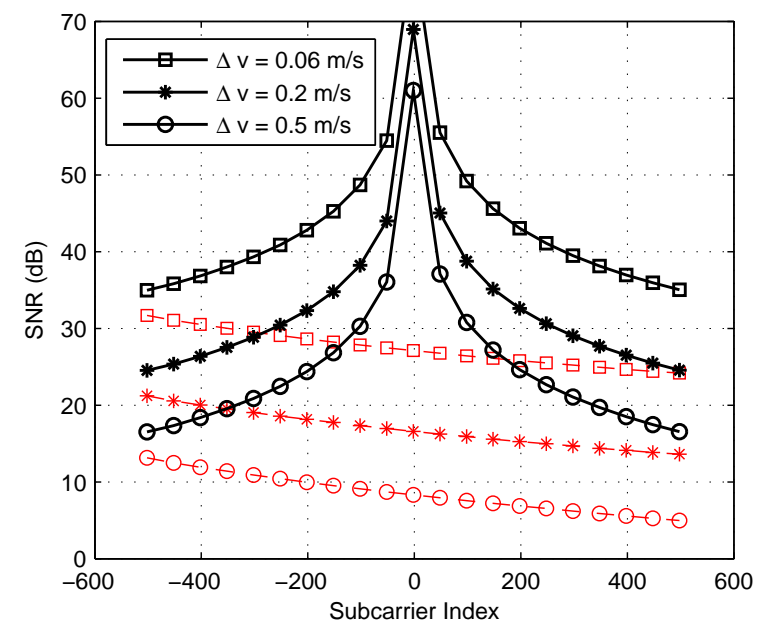

Fig. 4. The SNR upperbound $\bar{\gamma}_{m}$ for $\epsilon=\epsilon_{\mathrm{opt}}$ (thick, full lines) and $\epsilon=0$ (thin, dashed lines) as a function of $\Delta v$, where $a-b=\Delta v / c$.

Fig. 4 provides guidelines on the selection of the Doppler scale spacing of the parallel correlators. For example, assuming that the correlator branch closest to the true speed will yield the maximum metric, then with fine Doppler shift compensation $\epsilon=\epsilon_{\mathrm{opt}}$ we can set the Doppler scale spacing to be $0.4 \mathrm{~m} / \mathrm{s}$ (where we need $\Delta v$ to be less than $0.2 \mathrm{~m} / \mathrm{s}$ ) to achieve an SNR upperbound of at least $25 \mathrm{~dB}$. On the other hand, if an SNR upperbound of $15 \mathrm{~dB}$ is sufficient, the Doppler scale spacing could be as large as $1.0 \mathrm{~m} / \mathrm{s}$.

\section{IMPLEMENTATION AND PERFORMANCE TESTING}

In Section IV, it was shown that for the preamble with $K_{0}=512$ and $B=12 \mathrm{kHz}$, a speed mismatch of up to $1.5 \mathrm{~m} / \mathrm{s}$ did not degrade the detection performance considerably. On the other hand, the SNR analysis for data reception with $K=$ 1024 and $B=12 \mathrm{kHz}$ in Section $\mathrm{V}$ indicated that the speed mismatch should not exceed $0.3-0.5 \mathrm{~m} / \mathrm{s}$ to limit ICI. This suggests a multi-grid approach for the implementation:

1) Coarse-grid search for detection. Only a few parallel self-correlators are used to monitor the incoming data. This helps to reduce the receiver complexity.

2) Fine-grid search for data demodulation. After a detection is declared, a set of parallel self-correlators with better Doppler scale resolution is used only on the captured preamble. The fine-grid search is centered around the Doppler scale estimate from the coarse-grid search.

Instead of a multi-grid search, one may also use an interpolation based approach to improve the estimation accuracy beyond the limit set by the step size. We borrow a technique from [10], which is usually used in spectral peak location estimation based on a limited number of DFT samples. After the coarse or fine-grid search, let $\left|X_{k}\right|$ denote the amplitude from the branch with the largest correlation output, and $\left|X_{k-1}\right|$ and $\left|X_{k+1}\right|$ are the amplitudes from the left and right

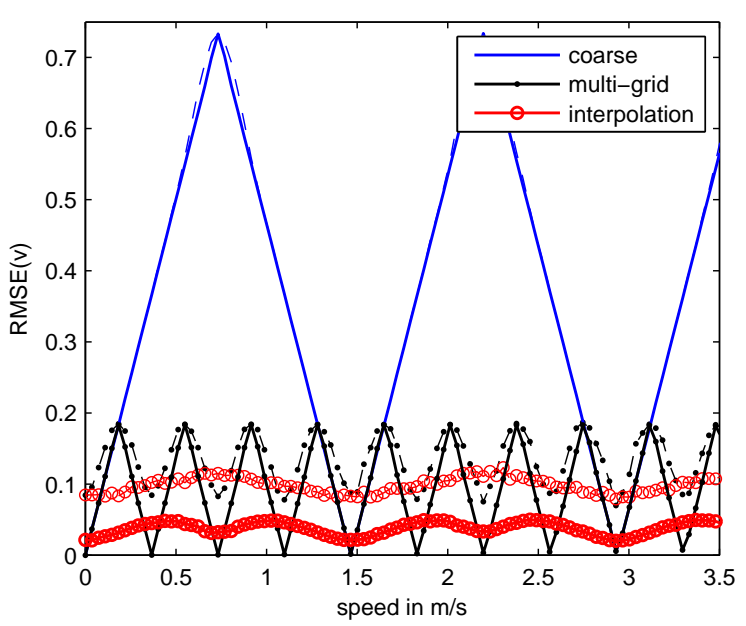

Fig. 5. Average velocity estimation error using varying amounts of correlators and a simple interpolation between the measured points; thin, dashed lines are $\gamma=0 \mathrm{~dB}$ and thick full lines are $\gamma=10 \mathrm{~dB}$.

neighbors. With $\Delta a$ as the grid spacing, we use

$$
\delta=\frac{\left|X_{k+1}\right|-\left|X_{k-1}\right|}{4\left|X_{k}\right|-2\left|X_{k-1}\right|-2\left|X_{k+1}\right|} \Delta a
$$

to estimate an offset $\delta$ of the Doppler scale deviating from the strongest branch towards the second strongest branch.

\section{A. Simulations for Velocity Estimation}

We set $K_{0}=512, B=12 \mathrm{kHz}$, and an oversampling factor of $\lambda=8$. The coarse grid spacing is set as $\Delta a=$ $\Delta v / c$, where $\Delta v$ is $1.46 \mathrm{~m} / \mathrm{s}$. Fig. 5 depicts the root mean square error of the speed estimates $\hat{v}=c \hat{a}$, at two SNRs of $0 \mathrm{~dB}$ and $10 \mathrm{~dB}$. We observe a "saw-tooth" shape for the coarse estimates, and the SNR decrease has little impact on this shape. This suggests that the probability of not finding the closest branch is negligible and the dominating error is the quantization to the coarse grid.

After detection of the coarse-grid search, we use another six self-correlators with spacing of $\Delta v=0.366 \mathrm{~m} / \mathrm{s}$ to search around the estimated Doppler scale from the previous stage. Much improved estimates are obtained, as shown in Fig. 5. The achieved accuracy exceeds the mismatch specification we set earlier of 0.3-0.5 m/s. More degradation of the sawtooth shape shows up at low SNR, which is reasonable. As the separation in tentative Doppler scales between correlators diminishes, neighboring correlators will have very similar outputs. Fig. 5 also shows the RMSE for velocity estimation with interpolation after the coarse grid search with $\Delta v=1.46 \mathrm{~m} / \mathrm{s}$. We observe that the interpolation approach is very effective.

\section{B. Results with Experimental Data}

We now work on the data from an experiment performed at Buzzards Bay, Dec. 15, 2006 with a fast-moving transmitter. The same data set was used previously in [7] to demonstrate the capability of OFDM reception in a dynamic setup, where Doppler scale estimation was done based on the measured time 


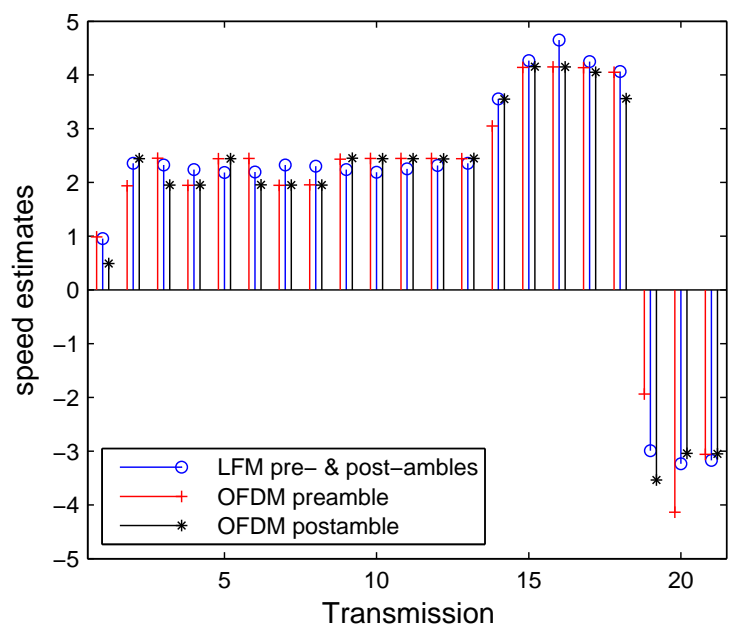

Fig. 6. Comparison of velocity estimation techniques; the previous method calculated the time difference between a LFM pre- and postamble, while the new approach is solely based on one packet.

difference between the LFM pre- and postamble. The lengths of the transmitted packets in this experiment were fairly long [7]. For example, with $K=1024$, a total of 32 OFDM blocks are inside one packet, which lasts lasts about 3.53 seconds since each OFDM block has $T=85.3 \mathrm{~ms}$ and $T_{g}=25 \mathrm{~ms}$ in this case.

In addition to the LFM pre- and post-ambles, an OFDM preamble and an OFDM postamble were also transmitted along each packet. In Fig. 6 we plot the relative velocity estimates for three settings: i) using the LFM preamble and postamble, ii) using the OFDM preamble only, and iii) using the OFDM postamble only. We observe that the speed estimates are close to each other. The estimates from the proposed method differ from those of the previous method by no more than 1 knot $(\approx 0.5 \mathrm{~m} / \mathrm{s})$ for any transmission. It is worth pointing out that the LFM method uses the duration of a complete transmission while the proposed method yields an estimate based on a single block.

We next carry out a comparison on the BER performance, where QPSK modulation and a 16-state rate $2 / 3$ convolutional code was used in the data set [7]. Demodulation and decoding were done twice for each packet transmission; once using the Doppler scale estimate obtained from the LFM method and once using the estimate based on the OFDM preamble only. Fig. 7 shows that similar uncoded and coded BER results are obtained. The proposed method, however, avoids the need of buffering the whole packet before data demodulation.

\section{CONCLUSION}

In this paper we proposed a new method for detection, synchronization, and Doppler scale estimation for underwater acoustic communication based on multicarrier waveforms. We characterized the receiver operating characteristics and analyzed the impact of Doppler scale estimation accuracy on the system performance. With real data, we verified that the

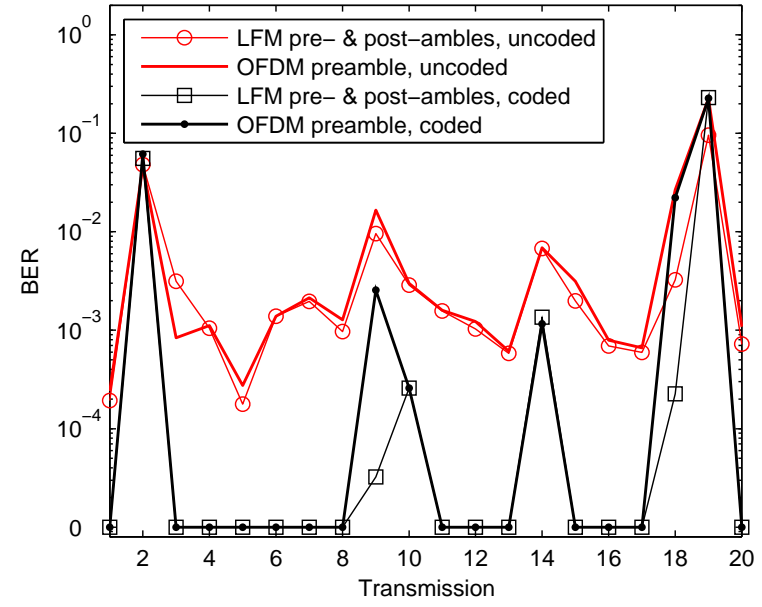

Fig. 7. Comparison of the uncoded and coded bit error rates; decoding after resampling with either the offline speed estimates based on the LFM pre- and postambles or based on the new speed estimate for online processing.

performance of the proposed method on Doppler scale estimation is comparable to a previous method based on the LFM preamble and postamble. The key advantage of the proposed method is that it avoids the need to buffer the whole packet before data demodulation. Hence, it is very appealing for the development of online receivers for multicarrier underwater acoustic communication.

\section{REFERENCES}

[1] D. B. Kilfoyle and A. B. Baggeroer, "The state of the art in underwater acoustic telemetry," IEEE J. Ocean. Eng., vol. 25, no. 1, pp. 4-27, Jan. 2000.

[2] T. M. Schmidl and D. C. Cox, "Robust frequency and timing synchronization for OFDM," IEEE Trans. Commun., vol. 45, no. 12, pp. 16131621, Dec. 1997.

[3] H. Minn, V. Bhargava, and K. Letaief, "A robust timing and frequency synchronization for OFDM systems," IEEE Trans. Wireless Commun., vol. 2, no. 4, pp. 822-839, July 2003.

[4] R. D. J. van Nee, G. A. Awater, M. Morikura, H. Takanashi, M. A. Webster, and K. W. Halford, "New high-rate wireless LAN standards," IEEE Communications Magazine, vol. 37, no. 12, pp. 82-88, Dec. 1999.

[5] M. Stojanovic, "Low complexity OFDM detector for underwater channels," in Proc. of MTS/IEEE OCEANS conference, Boston, MA, Sept. 18-21, 2006.

[6] B. Li, S. Zhou, M. Stojanovic, and L. Freitag, "Pilot-tone based ZPOFDM demodulation for an underwater acoustic channel," in Proc. of MTS/IEEE OCEANS conference, Boston, MA, Sept. 18-21, 2006.

[7] B. Li, S. Zhou, M. Stojanovic, L. Freitag, and P. Willett, "Non-uniform Doppler compensation for zero-padded OFDM over fast-varying underwater acoustic channels," in Proc. of MTS/IEEE OCEANS, Aberdeen, Scotland, June 2007.

[8] J. H. Conway, R. H. Hardin, and N. J. A. Sloane, "Packing lines, planes, etc.: Packings in Grassmannian space," Experimental Math., vol. 5, no. 2, pp. 139-159, 1996.

[9] S. Mason, C. R. Berger, S. Zhou, and P. Willett, "Detection, synchronization, and Doppler scale estimation with multicarrier waveforms in underwater acoustic communication," IEEE J. Select. Areas Commun., Feb. 2008, submitted for publication.

[10] E. Jacobsen and P. Kootsookos, "Fast, accurate frequency estimators [DSP Tips \& Tricks],” IEEE Signal Processing Magazine, vol. 24, no. 3, pp. 123-125, May 2007. 